\title{
Infective Endocarditis at Tricuspid Valve in CHD: What are its Characteristics? What is the Pathophysiology?
}

\section{Boussir $\mathrm{H}^{*}$, Bachrif M, Fliti A, El ouafi $\mathrm{N}$ and Ismaili $\mathrm{N}$}

Department of Cardiology, University Hospital of Mohammed VI, University Mohammed the First, Oujda, Morocco

${ }^{*}$ Corresponding author: Boussir H, Department of Cardiology, University Hospital of Mohammed VI, Oujda University, 60049.PB 4806 Oujda, Morocco, Tel: 00212610025247, E-mail: hanane0802@gmail.com

Citation: Boussir H, Bachrif M, Fliti A, El ouafi N, Ismaili N (2017) Infective Endocarditis at Tricuspid Valve in CHD: What are its Characteristics? What is the Pathophysiology? J Case Rep Stud 5(3): 302. doi: 10.15744/2348-9820.5.302

Received Date: June 08, 2017 Accepted Date: June 27, 2017 Published Date: June 29, 2017

\begin{abstract}
Infective endocarditis (IE) is a serious infectious disease that carries a high mortality rate. We report the case of 74-year-old female who is a chronic hemodialysis patient, and was presented with tricuspid valve endocarditis (TVIE) with central venous catheter, due to an Enterobacter Cloacae, which is a non-HACEK Gram-negative bacilli (GNB) and has been reported to be an extremely rare pathogen of IE. The patient was treated with imipenem and teicoplanin for a four-week period, with negative Blood culture and normal $C$ reactive protein $(C R P)$ levels at the end. The aim of our study is to understand the pathophysiology of the IE in chronic renal failure (CRF) especially at the tricuspid valve (TV), and to determine the clinical, biological characteristics and therapeutic modalities.
\end{abstract}

Keywords: Infective Endocarditis; Chronic Hemodialysis; Tricuspid Valve

\section{Introduction}

Infective endocarditis (IE) has been recognized as a complication of hemodialysis since 1966. Since then, several studies have been published of relatively small series of patients [1].

The pathophysiology of IE in chronic hemodialysis (CHD) is complex and not completely understood. It is based mainly on the cardiac valvular and perivalvular abnormalities classically found at an early stage of chronic renal failure (CRF).

It is questionable to apply the Duke criteria in their strictest form to hemodialysis (HD) patients, since they could underdiagnose IE and significantly delay the time to diagnosis in these patients: many HD patients have a vascular access device in situ and hence a potential primary focus of infection precluding bacteraemia from being a major criterion; fever, another component of the Duke criteria, is present less commonly in HD patients (45-70\%) than in the general population; other signs commonly accompanying an infectious disease in the general population are not helpful in this subset of patients; some of these, such as increased erythrocyte sedimentation and anaemia, are already present in the end-stage renal disease (ESRD), whereas others, such as haematuria, may be specifically absent.

Also, in CHD, IE of the right heart remains rare and its frequency varies from $0 \%$ to $26 \%$ depending on the study [1]. The tricuspid valve (TV) is the main location, while involvement of the pulmonary valve is exceptional $[2,3]$.

Antibiotic therapy is the primary treatment strategy for isolated tricuspid valve endocarditis (TVIE) with less than $5 \%$ mortality rate. However; surgery is preferred for patients in whom medical therapy has failed.

Gram-positive cocci such as Streptococcus spp. and Staphylococcus spp. are mainly responsible for infective endocarditis. In contrast, Enterobacter spp, a non-HACEK GNB, has been reported to be an extremely rare pathogen of IE, and IE due to non-HACEK GNB including Enterobacter cloacae is known to be highly severe.

We present the survival case of IE due to E. cloacae at the TV in a 74-year-old female who is a chronic hemodialysis patient with central venous catheter.

\section{Case report}

A 74-year-old female with a history of type 2 diabetes mellitus for 20 years, high blood pressure for 13 years, and chronic hemodialysis for 9 years currently with central venous catheter for more than one year, because the achievement of the arteriovenous fistula 
(AVF) failed, was admitted to hospital because of fever and chills only at the moment of hemodialysis for more than 3 months. On physical examination, his temperature was $37^{\circ} \mathrm{C}$, his heart rate was $80 \mathrm{bpm}$ and the respiratory rate was 16 breaths per minute. The patient's blood pressure was 130/80 mmHg. A systolic murmur, grade 2 over 6 , was heard at the tricuspid valve site. The lungs were clear bilaterally. Peripheral stigmata of IE were absent.

Laboratory findings included Hemoglobin of $8 \mathrm{~g} / \mathrm{dl}$, a White blood cell count of $10000 / \mathrm{mm} 3$, an Erythrocyte sedimentation rate of $39 \mathrm{~mm}$ /hour. C reactive protein (CRP) was in the range $98 \mathrm{mg} / \mathrm{l}$. Urine cytology and Culture results were negative. Plain chest radiography was reported normal.

On the fifth day, we received a report that three Blood cultures were positive for an Enterobacter Cloacae. The prescribed antibiotics (cloxacillin and gentamycin) were changed to imipenem and teicoplanin after antibiogram, and the jugular catheter was removed and replaced by femoral catheter. High-resolution computed tomography (HRCT) of the whole body and the fund of eye were normal.

Transthoracic echocardiography (TTE) showed a severe tricuspid valve regurgitation (Figure 1), that is the result of the TV perforation (Figure 2), and also showed a perivalvular lucent space (abscess) of the TV (Figure 3), without vegetation. A transesophageal cardiac echocardiography could not be made.

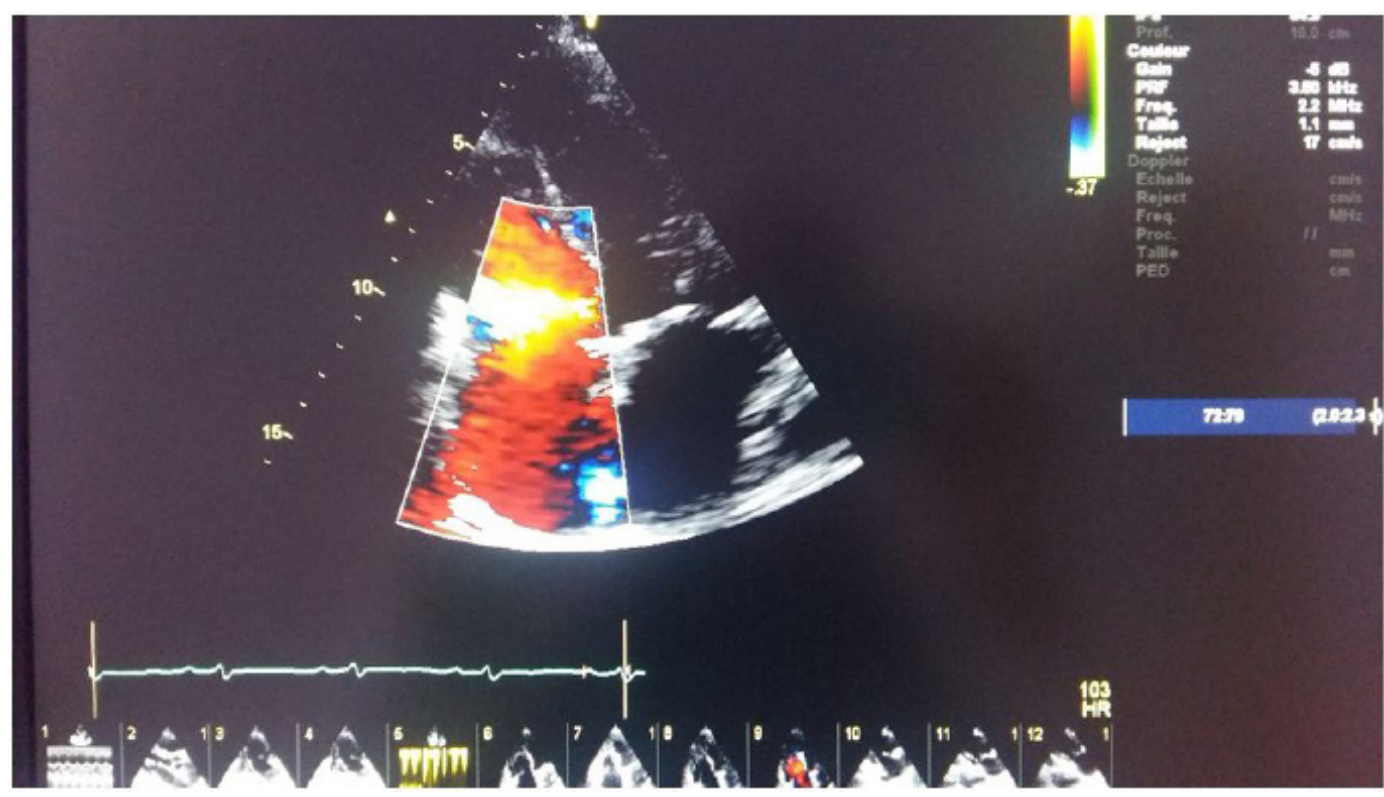

Figure 1: Color Doppler imaging demonstrating severe tricuspid valve regurgitation

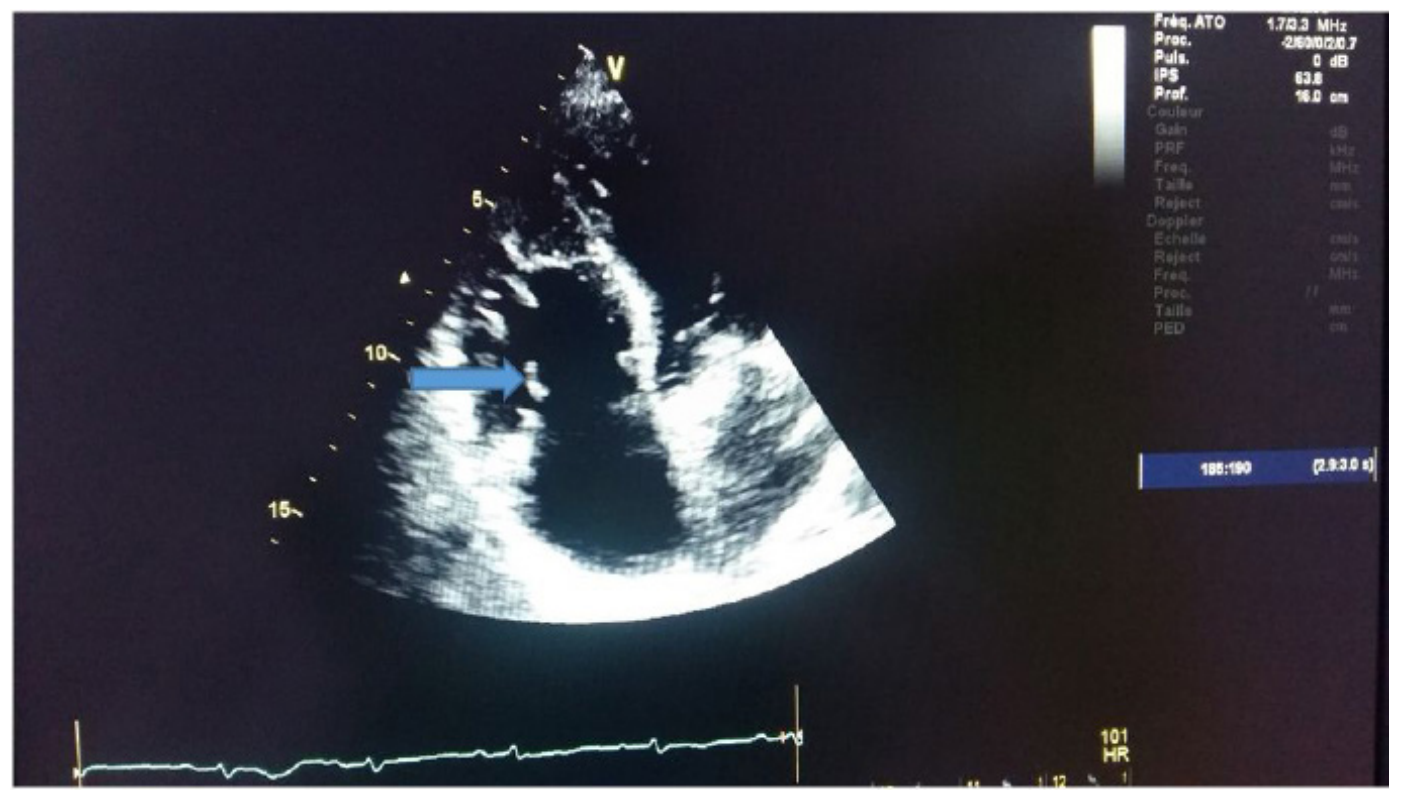

Figure 2: Transthoracic echocardiography: an apical four-chamber view showing a tricuspid valve perforation with a dilated right atrium and right ventricle 




Figure 3: Modified long axis view shows a perivalvular lucent space (abscess) of the tricuspid valve

The patient was treated with imipenem and teicoplanin for a four-week period, with negative Blood culture and normal CRP levels at the end. She is waiting for surgery which is indicated for severe TV regurgitation after perforation and for abscess.

\section{Discussion}

The risk of IE in ESRD patients is significantly higher than in the general population. There are several potential explanations for the increased incidence of IE in HD patients:

Patients with ESRD have an increased incidence of degenerative heart valve disease, which is a major risk factor for IE [4]. Calcific aortic stenosis [4], mitral annular calcification with consequent mitral regurgitation, and/or stenosis [4] and bioprosthetic valve degeneration [5] are extremely frequent in this group of patients. Furthermore, degenerative heart valve disease is premature since it appears to begin 10-20 years earlier than in the general population [6]. The accelerated development of valvular calcification in ESRD patients is thought to be related to the abnormalities of calcium-phosphorus homeostasis in the setting of secondary hyperparathyroidism and to the chronic micro-inflammatory milieu of uraemia associated with ESRD [4]. These abnormalities are more pronounced when the duration of CRF and dialysis is long [7] as seemed to occur in our case.

Episodes of bacteraemia during HD are relatively common; they develop at an estimated rate of one episode per 100 patient-care months $[8,9]$. They are primarily the result of frequent intravascular access through arteriovenous fistula (AVF), vascular graft, or indwelling vascular catheter [9] and may originate from either endogenous (i.e. patient's own cutaneous flora, the major cause of staphylococcal infections in these patients) or exogenous sources (i.e. hands of personnel, contaminated equipment) [10]. which joined the case of our patient, who had a tunneled catheter since more than one year because she presented with ischemia of the fingers caused by the AVF.

Patients with ESRD are inherently prone to bacteraemia and IE also as a result of an impairment of the immune system. Metabolic abnormalities associated with ESRD, malnutrition, and associated comorbidities, such as diabetes mellitus, as seemed to occur in our case, may indeed impair polymorphonuclear cell function and granulocyte mobility, reducing cellular host defence and clearance of bacteria from the bloodstream [9].

Staphylococcus aureus is the main cause of vascular access-related bacteraemia among patients receiving longterm HD (up to $75 \%$ of cases) [11], contrary to our patient who had an Enterobacter Cloacae, which is a non-HACEK Gram-negative bacilli (GNB). There are few reported cases of IE due GNB [12]. In these rare GNB IE cases, groups of GNB consisting of Haemophilus, Actinobacillus, Cardiobacterium, Eikenella, and Kingella spp. (HACEK) are the predominant cause [12]. In contrast, Enterobacter $s p p$, a non-HACEK GNB, has been reported to be an extremely rare pathogen of IE. Gut translocation of E. cloacae may have caused bacteremia and finally IE in our patient; however we were unable to identify its site of entry [13].

The clinical presentation of IE in the HD population is often difficult to distinguish from that of an uncomplicated access infection [11]. Furthermore, the diagnosis of IE in HD patients using the Duke criteria $[14,15]$ could be problematic. The use of Duke criteria in this group of patients has indeed some limitations; first, they require the presence of bacteraemia in the absence of a removable focus of infection for diagnosing IE. However, many HD patients have a vascular access device in situ and hence a potential primary focus of infection precluding bacteraemia from being a major criterion $[2,8,16]$. Second, fever, another component of the Duke criteria, is present less commonly in HD patients (45-70\%) than in the general population (80-90\%), probably due 
to uraemia-related impaired cellular host defence [16]. Although the absence of fever has high negative predictive value for a diagnosis of IE in the general population [14] it is not a useful diagnostic feature in HD patients. Therefore, it is questionable to apply the Duke criteria in their strictest form to HD patients, since they could underdiagnose IE and significantly delay the time to diagnosis in these patients. In addition to this, fever and lung impairment are the main signs of IE of the right heart, what is missing in our patient, Which explain the diagnostic delays as shown by the complications in echocardiography : perforation and abscess of the tricuspid valve.

Furthermore, other signs commonly accompanying an infectious disease in the general population are not helpful in this subset of patients; some of these, such as increased erythrocyte sedimentation and anaemia, are already present in ESRD, whereas others, such as haematuria, may be specifically absent [17]. These considerations further emphasize the importance of echocardiography in the assessment of HD patients with bacteraemia and suspected IE.

As was already mentioned above, the IE of the right heart in CHD is rare. The tricuspid and pulmonary valves do not undergo change during CRF because they are in a low-pressure cardiac system. Bacterial overgrowth during IE thus occurs more easily on the cardiac valves previously damaged, explaining the high prevalence of involvement of left-sided valves and the rarity of rightsided valvular involvement during IE in CHD.

Why our patient had IE at the right heart, especially at the TV? The predominant involvement of the tricuspid valve is not yet completely understood in this context. Might the reason for this finding be related to the location of this valve? It is the first valvular line of contact with the blood flow arriving from the vena cava and massively enriched with bacterial particles [1], in addition to that, the catheter of our patient was jugular, located near the TV, this means direct contact of the valve with bacterial particles.

Staphylococcus aureus or coagulase-negative Staphylococcus species (Gram-positive cocci) are the common cause of right sided IE. $70 \%$ to $85 \%$ of the right sided IE can be treated by medical therapy. Our patient had an Enterobacter Cloacae which is a non HACEK GNB. Some specialists recommend that cases of IE associated with non-HACEK GNB should be managed by early surgery, combined with long-term ( $>6$ weeks) therapy consisting of combinations of $\beta$-lactams and aminoglycosides, others have reported that the mortality rate is identical in surgical and non surgical cases as well as in patients treated with dual- as opposed to single-antibiotics [18]. Our patient was treated by Imipenem and teicoplanin for a four-week period and the jugular catheter was removed, and replaced by a femoral catheter, with negative Blood culture and normal CRP levels at the end. She is at the time of writing, waiting for surgery which is indicated for severe TV regurgitation after perforation and for abscess. The advanced age and vulnerability of our patient as well as the good evolution under antibiotics prevents us from making a surgical decision and fixing an intervention date until now. Yoshino et al. reported a clone of E.cloacae, which was resistant to third-and fourth-generation cephalosporins and was successfully treated using a combination of meropenem and amikacin for 12 weeks and no surgical intervention was made [19]. This approach approximate that adopted in our case.

\section{Conclusion}

The clinical and biological diagnosis of IE in CHD can be difficult, which emphasize the importance of echocardiography.

Strict respect of aseptic procedures during any manipulation of the vascular access and early detection of any infection of the vascular access continue to offer the only sure approaches to reduce the incidence of IE in CHD.

\section{References}

1. Bentata Y, Haddiya I, Ismailli N, El Ouafi N, Benzirar A, et al. (2016) Infective Endocarditis in Chronic Hemodialysis: A Transition from Left Heart to Right Heart. Saudi J Kidney Dis Transpl 27: 1200-6.

2. Doulton T, Sabharwal N, Cairns HS, Schelenz S, Eykyn S, et al. (2003) Infective endocarditis in dialysis patients: new challenges and old. Kidney Int 64: $720-7$.

3. Rekik S, Trabelsi I, Hentati M, Hammami A, Jemaa MB, et al. (2009) Infective endocarditis in hemodialysis patients: clinical features, echocardiographic data and outcome: a 10-year descriptive analysis. Clin Exp Nephrol 13: 350-4.

4. Umana E, Ahmed W, Alpert MA (2003) Valvular and perivalvular abnormalities in end-stage renal disease. Am J Med Sci 325: 237-42.

5. Fishbein MC, Gissen SA, Collins JJ Jr, Barsamian EM, Cohn LH (1977) Pathologic findings after cardiac valve replacement with glutaraldehyde-fixed porcine valves. Am J Cardiol 40: 331-7.

6. Madu EC, D'Cruz IA, Wall B, Mansour N, Shearin S (2000) Transesophageal echocardiographic spectrum of calcific mitral abnormalities in patients with endstage renal disease. Echocardiography 17: 29-35.

7. Ellouali F, Berkchi F, Elhoussni S, Bayahia R, Benamar L, et al. (2015) Evaluation of the effect of duration on dialysis on echocardiographic parameters: a preliminary study. Saudi J Kidney Dis Transpl 26: 83-9.

8. McCarthy JT, Steckelberg JM (2000) Infective endocarditis in patients receiving long-term hemodialysis. Mayo Clin Proc 75: 1008-14.

9. Powe NR, Jaar B, Furth SL, Hermann J, Briggs W (1999) Septicemia in dialysis patients: incidence, risk factors, and prognosis. Kidney Int 55: 1081-90.

10. Sexton DJ (2001) Vascular access infections in patients undergoing dialysis with special emphasis on the role and treatment of Staphylococcus aureus. Infect Dis Clin North Am 15: 731-42.

11. Maraj S, Jacobs LE, Maraj R, Kotler MN (2004) Bacteremia and infective endocarditis in patients on hemodialysis. Am J Med Sci 327: $242-9$. 
12. Baddour LM, Wilson WR, Bayer AS, Fowler Jr VG, Bolger AF, et al. (2005) Infective endocarditis: diagnosis, antimicrobial therapy, and management of complications: a statement for healthcare professionals from the Committee on Rheumatic Fever, Endocarditis, and Kawasaki Disease, Council on Cardiovascular Disease in the Young, and the Councils on Clinical Cardiology, Stroke, and Cardiovascular Surgery and Anesthesia, American Heart Association: endorsed by the Infectious Diseases Society of America. Circulation 111: e394-434.

13. Wiest R, Garcia-Tsao G (2005) Bacterial translocation (BT) in cirrhosis. Hepatology 41: 422-33.

14. Durack DT, Lukes AS, Bright DK (1994) New criteria for diagnosis of infective endocarditis: utilization of specific echocardiographic findings. Duke Endocarditis Service. Am J Med 96: 200-9.

15. Li JS, Sexton DJ, Mick N, Nettles R, Fowler VG Jr, et al. (2000) Proposed modifications to the Duke criteria for the diagnosis of infective endocarditis. Clin Infect Dis 30: 633-8.

16. Maraj S, Jacobs LE, Kung SC, Raja R, Krishnasamy P, et al. (2002) Epidemiology and outcome of infective endocarditis in hemodialysis patients. Am J Med Sci 324: 254-60.

17. Vijayvargiya R, Veis JH (1996) Antibiotic-resistant endocarditis in a hemodialysis patient. J Am Soc Nephrol 7: 536-42.

18. Morpeth S, Murdoch D, Cabell CH, Karchmer AW, Pappas P, et al. (2007) Non-HACEK gram-negative bacillus endocarditis. Ann Intern Med 147: 829-35.

19. Yoshino Y, Okugawa S, Kimura S, Makita E, Seo K, et al. (2015) Infective endocarditis due to Enterobacter cloacae resistant to third- and fourth-generation cephalosporins. J Microbiol Immunol Infect 48: 226-8.

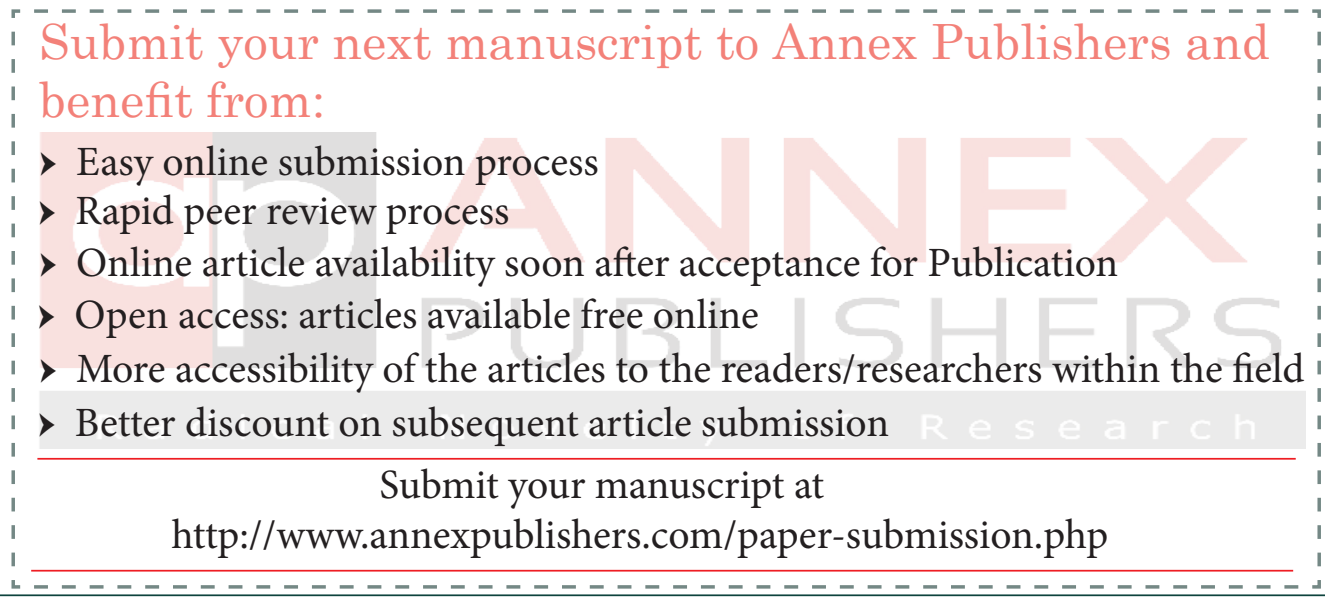

